\title{
Determining Woody-to-Total Area Ratio using Terrestrial Laser Scanning (TLS)
}

Lixia Ma ${ }^{1,2}$, Guang Zheng ${ }^{2 *}$, Jan U. H. Eitel ${ }^{3,4}$, Troy S. Magney ${ }^{5}$, L. Monika Moskal ${ }^{6}$

${ }^{1}$ Jiangsu Center for Collaborative Innovation in Geographical Information Resource Development and Application, Nanjing, 210023, China

${ }^{2}$ Jiangsu Provincial Key Laboratory of Geographic Information Science and Technology, International Institute for Earth System Science, Nanjing University, China, 210023

${ }^{3}$ Geospatial Laboratory for Environmental Dynamics, University of Idaho, Moscow, ID 83844-1135, USA

${ }^{4}$ McCall Outdoor Science School, University of Idaho, McCall, ID 83638, USA

${ }^{5}$ NASA Jet Propulsion Laboratory, 4800 Oak Grove Dr. MS 233-300, Pasadena, CA, 91109

${ }^{6}$ Remote Sensing and Geospatial Analysis Laboratory, Precision Forestry Cooperative, School of Environment and Forest Science, University of Washington Box 352100, Seattle, Washington, USA, 98115-2100 


\section{Abstract}

Accurately determining woody-to-total area ratio (WTA) is a key step to indirectly retrieve leaf area index (LAI) from terrestrial laser scanning (TLS) data. In this work, we first collected both individual tree and forest plot point cloud data (PCD) from broadleaf- and coniferous tree species and leaf characteristics using both side-lateral and full field-of-view TLS field setups with scan distances between 2.5 to 28 meters. Using a local geometrical feature-based algorithm, the generated PCD were automatically classified into three different categories including photosynthetic canopy components, non-photosynthetic canopy components, and bare earth. To convert each classified point into a surface area, we then developed and validated a novel approach that considers sampling space, laser incidence angle, and leaf orientation information. The estimated surface areas from this approach showed strong agreements with validation datasets for single leaf (91.44\%), photosynthetic (95.64\%), and non-photosynthetic canopy components $(89.60 \%)$ of an artificial tree and stems of an old-growth coniferous tree $(93.53 \%)$, two individual broadleaf trees $(98.31 \%$ and $97.46 \%)$ and a broadleaf forest plot $(90.26 \%)$. By doing this, we computed the parameter WTA for an individual artificial tree $(10.90 \%)$, an old-growth coniferous tree (29.97\%), two individual broadleaf tree $(14.83 \%$ and $4.27 \%)$ and four natural forest stands ranging from $7.74 \%$ - $15.57 \%$, respectively. The proposed method can effectively improve the accuracy of retrieving true LAI by removing the effects of woody components and converting each point into a surface area.

Key words: terrestrial lidar, woody-to-total area ratio, leaf area index (LAI), sampling space, leaf orientation 


\section{Introduction}

Leaf area index(LAI), defined as one half of the total green leaf area per unit ground surface area, is a primary control on the exchange of energy and mass exchanges between the atmosphere and terrestrial ecosystems (Amthor et al., 1990; Brümmer et al., 2012; Chasmer et al., 2008). Both direct and indirect methods can be used to measure LAI (Norman, 1989). The labor-intensive and time consuming nature of direct-based method (i.e., destructive sampling-based measurement) limits its use across broader spatial scales. In contrast, indirect- methods can be used across broader spatial scales but challenges remain; for example, it is very difficult to acquire the three dimensional (3-D) structural information of forest canopies from traditional optical approach such as digital hemispherical photography (DHP). Since only green photosynthetic canopy components are considered in LAI retrievals, the proportions of green photosynthetic and woody canopy components of an individual tree or forest stand should be quantitatively characterized (Breda, 2003). Some research suggests that woody canopy components are a major source of error in indirect- LAI estimates (Béland et al., 2014; Whitford et al., 1995), and non-photosynthetic components can account for about $4.2 \%$ $-32.7 \%$ of leaf area density estimation among various tree species and stand ages (Hosoi and Omasa, 2007). The accurate quantification of the contribution of woody materials (i.e., non-photosynthetic canopy components) is hence a key step to further improve the accuracy of indirect LAI estimates.

The proportion of woody materials of an individual tree or forest stand can be characterized by the parameter commonly referred to as the "woody-to-total area ratio" (WTA) defined as the ratio of woody surface area and total surface area of an individual 
tree or forest stand (Zou et al., 2009). To determine WTA, spectral information may permit the optical separation of photosynthetic and non-photosynthetic canopy components. For example, the multiband vegetation imager (MVI) has been used to determine WTA (Kucharik et al., 1998a; Kucharik et al., 1998b; Kucharik et al., 1997; Zou et al., 2009). However, these passive optical-based approaches have some limitations: (1) it is difficult to image canopy components in the upper part of taller forest canopies (Kucharik et al., 1997) ; (2) within canopy shadowing and inappropriate exposure settings of images will introduce errors to obtain final WTA and ; (3) they only permit capturing images of canopies in two-dimensions (2-D), thereby limiting their ability to vertically resolve different canopy components.

In contrast to traditional passive optical approaches, point cloud data (PCD) generated from light detection and ranging (LiDAR) provide 3-D structural information of forest canopies (Alonzo et al., 2014; Antonarakis et al., 2010; Lefsky et al., 1999; Roberts et al., 2005; Zheng et al., 2013). In particular, terrestrial laser scanning (TLS) enables generating highly spatially resolved $(<5 \mathrm{~mm}$ point sampling space) $\mathrm{PCD}$ to vertically resolve forest structural information in great detail such as vertical plant profiles (Calders et al., 2015b), leaf orientation (Eitel et al., 2010; Zhao et al., 2015; Zheng and Moskal, 2012), and forest aboveground biomass (Calders et al., 2015a; Stovall and Shugart, 2014). There are three different types of information contained within the TLS-based PCD including the $\mathrm{x}, \mathrm{y}, \mathrm{z}$ geometric information, laser return intensity information (LRI), and red, green, and blue (RGB) color information. Because the complex light illumination condition within a forest stand, the RGB color information is difficult to utilize even with correct exposure setting (Ma et al., 2016). Though the LRI 
information has been used to separate photosynthetic from non-photosynthetic canopy components (Béland et al., 2014; Cook and Chakrabarti, 2015; Danson et al., 2014; Douglas et al., 2015), it remains challenging to reliably classify canopy components based on LRI due to the strong incidence angle effect on the strength of the returning laser signal (Eitel et al., 2014; Magney et al., 2014).

Over the last few years, several groups developed automatic methods to classify forest PCD based on only geometric, or the combination of geometric and color / intensity information (Cote et al., 2009; Lalonde et al., 2006; Raumonen et al., 2013; Vandapel et al., 2004; Yang et al., 2013). Recently, Ma et al. (2016) developed and validated an automated point classification method to separate photosynthetic and non-photosynthetic canopy components solely based on geometric information contained within the TLS data. However, converting the classified points into a surface area and ultimately WTA remains challenging due to factors such as incident angle of laser beam, foliage orientation, and the relative distances and heights between TLS and target objects that affects the spatial distribution pattern and density of the collected PCD. The overarching goal of this work was to develop a robust and reliable approach for determining WTA from TLS data. Our specific objectives were to:

1) Classify forest PCD into photosynthetic canopy components, non-photosynthetic canopy components, and bare earth based on local geometric features, and evaluate the resulting classification accuracy;

2) Develop and validate a method to automatically compute WTA by converting each classified point into a surface area; and

3) Assess the effects of sampling space, laser incidence angle, and foliage 
orientation on TLS derived WTA estimates.

\section{Materials and Methods}

2.1 Point cloud data collection and pre-processing

To include a range of different leaf characteristics in our study, we collected PCD from broadleaf- and coniferous tree species (Fig. 1). To acquire the PCD, individual trees and forest plots were scanned from distances ranging from 5 to 28 meters using the different field acquisition methods including side-lateral and full field-of-view (FOV) (Horizontal: $0^{\circ} \sim 360^{\circ}$, Vertical: $-45^{\circ} \sim 90^{\circ}$ ) TLS setups (Fig. 2). All PCD were collected using a Leica Scan Station 2 (Leica Geosystem AG, St, Gallen, Switzerland) and technical characteristics of the Scan Station 2 are given in table 1. Information about different leaf characteristics and detailed information about three forest plots can be found in Table 2 .

\section{Please insert Figure 1 here.}

\section{Please insert Table 1 and 2 here.}

\subsubsection{Side-lateral scanning technique}

In October 2009, an old-growth Douglas fir (Pseudotsuga menziesii) tree at the Wind River Experimental Forest (WREF), Washington, USA was scanned from one side-lateral scanning location at a distance of $4 \mathrm{~m}$ away from TLS (Fig. 2a). The predefined sampling space was set as $10 \mathrm{~mm}$ at a $10 \mathrm{~m}$ distance from the TLS, and the mean neighbor point distance (MNPD), defined as the average distance between two regular sampled neighbor points, was $6 \mathrm{~mm}$. The tree height, dimeter at breast height (DBH), and crown size were about $42 \mathrm{~m}, 0.75 \mathrm{~m}$, and $10 \mathrm{~m}$, respectively. 
We also collected one individual Chinese aspen (Populus adenopoda) tree and forest plot PCD in the BaiMa (BM) research and experimental forest in Nanjing, China $\left(119.18552^{\circ} \mathrm{E}, 31.6142^{\circ} \mathrm{N}\right)$ between August 1st and 6th in 2015. Two side-lateral scan locations with opposite scanning directions of $5 \mathrm{~m}$ and $11.4 \mathrm{~m}$ distance from the target were setup for the individual tree to acquire a comprehensive tree PCD (Fig.2b). The sampling space was set to $10 \mathrm{~mm}$ at $10 \mathrm{~m}$ away from scanner with the MNPD set to 6 $\mathrm{mm}$. The tree height was about $23 \mathrm{~m}$ with an average leaf width of $110 \mathrm{~mm}$ (SD, standard deviation $=22 \mathrm{~mm})$ and length of $118 \mathrm{~mm}(\mathrm{SD}=29 \mathrm{~mm})$, respectively.

As shown in Fig. 2c, we built an artificial $2 \mathrm{~m}$ tall tree with a real stem and 487 artificial broadleaves. Two types of artificial broadleaves with the following dimensions were used: $0.15 \mathrm{~m}, 0.2 \mathrm{~m}$ (length) and $0.07 \mathrm{~m}, 0.09 \mathrm{~m}$ (width), respectively. The 3D properties of the tree were quantified with the Leica Scan Station 2. Under a well-controlled indoor environment, TLS scans were taken from five different scan locations with leaves on and off. All scan locations were located within a circle with a radius of $2.5 \mathrm{~m}$ and azimuthal angle intervals of $72^{\circ}$ between scans. Three additional targets were setup within the same circle with azimuthal angle intervals of $120^{\circ}$ to help registering the PCD. The predefined sampling space was set to $5 \mathrm{~mm}$ at $2.5 \mathrm{~m}$ with a MNPD of $5 \mathrm{~mm}$. Once the registration process was finished, a search sphere with a radius smaller than the predefined sampling space was used to iteratively go through every point of the registered tree PCD to remove redundant points within the search sphere. One of the two points was considered as a redundant point if the distance between these two points are smaller than the predefined sampling space, usually resulted from the registration error. Finally, we obtained two clean and comprehensive 
PCD for both leaf-on (i.e., whole PCD) and leaf-off (i.e., woody PCD) conditions. We then perfectly matched the 'whole PCD' and 'woody PCD' to remove the 'woody PCD' out from the 'whole PCD'. After doing this, we obtained a clean 'leaf PCD' out from 'whole PCD'. Due to the occlusion between foliage elements (Lovell et al., 2003), only partial surface of woody materials was usually sampled in the 'whole PCD'. By combining the 'leaf PCD' and 'woody PCD', we finally produced a comprehensive PCD covering all foliage elements for classification and computing WTA.

In addition, we scanned a natural individual broadleaf Camphor tree (Cinnamomum camphora) growing in the campus of Nanjing University (hereafter referred to "NanDa, ND”) on April 4th, 2016 (Fig. 2g). In order to obtain a relatively comprehensive tree PCD, we scanned the tree from two lateral sides with $7 \mathrm{~mm}$ predefined sampling spaces at the distances away from TLS at $25 \mathrm{~m}$ and $28 \mathrm{~m}$, respectively. To accompany this, one under-canopy full FOV TLS setup with a $7 \mathrm{~mm}$ sampling space at $1.5 \mathrm{~m}$ away from the tree stem was acquired. The average leaf length and width were $71 \mathrm{~mm}(\mathrm{SD}=18 \mathrm{~mm})$ and $34 \mathrm{~mm}(\mathrm{SD}=7 \mathrm{~mm})$, and the tree height and crown diameter were $7.5 \mathrm{~m}$ and $6 \mathrm{~m}$, respectively

\subsubsection{Full field-of-view scanning technique}

Two study sites with real trees were chosen to test and validate the proposed method.

The first forest plot study site was located in the Washington Park Arboretum (WPA) near Seattle, Washington, USA. In September 2008, hemispherical scans were taken from three forest plots with different densities (horizontal: $0^{\circ}-360^{\circ}$; vertical: $0^{\circ}-90^{\circ}$ ) (Fig. 2d). The dominant tree species of forest plot-1and plot-2 were Pacific madrone 
(Arbutus Menziesii), and Western red cedar (Thuja plicata) for forest plot-3. The sampling space was set to $100 \mathrm{~mm}$ at a distance of $30 \mathrm{~m}$ away from scanner and the MNPD was $50 \mathrm{~mm}$.

Lastly, two scan locations in the full-FOV TLS way were setup in a diameter line of the circular forest plot with the distance between two stations and radius of the forest plot as $10 \mathrm{~m}$ to acquire the forest PCD (Fig 2e) in the BM experimental forest. The dominant tree species in this forest plot is Goldenrain tree (Koelreuteria paniculata). The predefined sampling space and MNPD were $10 \mathrm{~mm} @ 5 \mathrm{~m}$ and $5 \mathrm{~mm}$, respectively. The mean tree height is $4.5 \mathrm{~m}$ with the leaf's width and length are $36 \mathrm{~mm}(\mathrm{SD}=7.4 \mathrm{~mm})$ and $74 \mathrm{~mm}(\mathrm{SD}=14 \mathrm{~mm})$, respectively.

\subsubsection{Individual artificial leaf}

To explore the effects of sampling space, incident angle of laser beams, and foliage elements' leaf orientation on the converted surface area of the artificial tree, we set up an additional controlled indoor experiment. A flat broadleaf was fixed at the same height as the TLS at a relative distance of $1 \mathrm{~m}$. We scanned the leaf with various combinations of sampling space and leaf inclination angles $(\theta)$ ranging from $0^{\circ}$ to $90^{\circ}$ at angular intervals of $15^{\circ}$ (Fig. 2f). For each fixed angle $\theta, 17$ different sampling spaces ranging from $2 \mathrm{~mm}$ to $90 \mathrm{~mm}$ at a $1 \mathrm{~m}$ distance from the TLS were used to obtain the artificial broadleaf PCD. The interval was set at $2 \mathrm{~mm}$ when sampling spaces ranged from $2 \mathrm{~mm}$ to $10 \mathrm{~mm}$, and at $5 \mathrm{~mm}$ and $10 \mathrm{~mm}$ for the sampling spaces ranging from $10 \mathrm{~mm}$ to 50 $\mathrm{mm}$, and $50 \mathrm{~mm}$ to $90 \mathrm{~mm}$, respectively.

\section{Please insert Figure 2 here.}




\subsection{Manually measured data}

We scanned two broadleaves of the artificial tree with different sizes and computed their surface area using the WinFOLIA software (Regent Instruments Inc. Quebec City, QC, Canada). With regard to woody materials, by assuming that stem and branches can be approximated by a cylinder, we divided the stems and branches of the artificial tree into small sections with a height of $0.2 \mathrm{~m}$, which can easily be manually measured. By measuring the diameter of the cross-sectional surface, we obtained the surface area for both of each cylindrical section for the stem or branch. Finally, the total woody material of the individual artificial tree can be computed by summing up all small sections of branches and stem.

\subsection{Computer-based mesh surface}

We meshed the 'leaf PCD' and 'woody PCD' using the Delaunay refinement paradigm algorithm provided in Geomagic studio (3D System. SC, USA) for the artificial tree. In addition, we reconstructed the partial sampled stem surfaces of the old-growth Douglas fir tree from WREF site, the individual broadleaf tree and forest plot from BM site and the individual broadleaf tree from ND site as validation data, respectively. The meshed surface was smoothed by minimizing angles between individual polygons provided by Geomagic studio (3D System. SC, USA). Based on the computer-based mesh results, we can compute the surface area for 'leaf PCD', 'woody PCD', or a combination of the two to obtain the total number of triangulated patches using the 'Cloud Compare' software package (version 2.6.2) [GPL software]. (2016). Retrieved from http://cloudcompare.org. 


\subsection{Point-wise classification}

Following approaches outlined in Ma et al. (2016), we used a geometric feature-based automatic forest point classification (GAFPC) algorithm to classify tree PCD into three different categories including photosynthetic, non-photosynthetic forest canopy components, and bare earth. The basic idea of the algorithm is that the PCD can be considered as a numeric matrix with the dimension as $\mathrm{N}$ by 3 , where $\mathrm{N}$ is the total number of points. Lalonde (1998) found that the ordered eigenvalues $\left(\lambda_{0} \geq \lambda_{1} \geq \lambda_{2}\right)$ of a given tree PCD can be used to characterize linear features $\left(\lambda_{0} \gg \lambda_{1} \approx \lambda_{2}\right)$ (i.e., non-photosynthetic canopy components), random features $\left(\lambda_{0} \approx \lambda_{1} \approx \lambda_{2}\right)$ (i.e., photosynthetic canopy components), and surface features $\left(\lambda_{0} \approx \lambda_{1} \gg \lambda_{2}\right)$ (i.e., bare earth) . Thus saliency feature $(S)$ can be expressed as (eqn.1):

$$
S=\left(\lambda_{2}, \lambda_{0}-\lambda_{1}, \lambda_{1}-\lambda_{2}\right)
$$

We first selected about 30 training samples for each of three classes, and computed their saliency features to build the Gaussian mixed models (GMM) of each class using expectation maximization (EM) algorithm. Then each point of a given tree PCD was put into three GMMs to compute the conditional probability belonging to one of the following three class: non-photosynthetic canopy components, photosynthetic canopy components, and surface features. Finally, each point was labeled as the class with highest conditional probability. After iteratively going through all points, we obtained the preliminary classification. Then, additional post-processing filters including edge filter, isolated filter, isolated density filter, ground filter, enhanced ground filter-1, and enhanced ground filter-2 were used to achieve the final best classification (Ma et al., 2016). 


\subsection{Conversion from point to surface}

The converted surface of each point varied with the predefined sampling spaces (Fig. 3a). We converted every point into surface area of both individual and forest plot PCD at three different levels including single leaf, photosynthetic and non-photosynthetic canopy components, respectively. The converted surface area were compared and validated with the manually measured and computer-based mesh results. The sampling space is determined by not only the transmission distance to target object of laser beam but also the horizontal distance between the origin and projected point of target in horizontal surface. Thus, we should compute the sampling space at a specific point for horizontal and vertical scales separately. As shown in Fig. 3b, $p_{i}$ is any point from foliage elements of a forest canopy, $p_{j}$ and $p_{k}$ are two points from the tree stem surface. The vertical sampling space only determined by the transmittance distance to target object of laser beams. Since the transmittance distances between all three points and the source point of laser beam and the vertical sampling space of all three points are the same (i.e., $\boldsymbol{O} \boldsymbol{p}_{i}=\boldsymbol{O} \boldsymbol{p}_{j}=\boldsymbol{O} \boldsymbol{p}_{k}$, and $g h=m n=s t$ ), and the horizontal sampling space of point $p_{j}$ and $p_{k}$ are the same $(C D=E F)$. However, the horizontal sampling space of the point $p_{i}$ can be computed according to its projected point $p_{i}^{\prime}$ since the horizontal sampling space is only related to horizontal distance. Although the transmittance distances of $p_{i}$ and $p_{j}$ are the same, their horizontal sampling space are different $(A B<$ $C D)$. The user predefined sampling space in both horizontal and vertical is $S_{h 0}$ and $S_{v 0}$ with the distance away from TLS in both directions as $D_{h 0}$ and $D_{v 0}$. Then, the quantitative relationship between sampling space in both horizontal and vertical directions $\left(S_{h}\right.$ and $\left.S_{v}\right)$ and distances away from TLS in both directions $\left(D_{h}\right.$ and $\left.D_{v}\right)$ can 
be expressed as (eqn. 2 and 3):

$$
\begin{aligned}
& \frac{S_{h}}{D_{h}}=\frac{S_{h 0}}{D_{h 0}} \\
& \frac{S_{v}}{D_{v}}=\frac{S_{v 0}}{D_{v 0}}
\end{aligned}
$$

where $S_{h 0}$, and $S_{v 0}$ are the predefined sampling space in horizontal and vertical directions at the distance $D_{h 0} D_{v 0}$; The predefined sampling space in both directions may be the same $\left(D_{h 0}=D_{v 0}\right)$. Thus, the corresponding horizontal and vertical sampling space with the distances between target and TLS as $D_{h}$ and $D_{v}$ can be computed as (eqn. 4 and 5):

$$
\begin{aligned}
& S_{h}=\left(S_{h o} \cdot D_{h} / D_{h 0}\right) \\
& S_{v}=\left(S_{v o} \cdot D_{v} / D_{v 0}\right)
\end{aligned}
$$

$D_{h}$ is the horizontal distance between the origin and projected point of target in the horizontal surface; $D_{v}$ is the transmittance distance of laser beam. Then the surface area represented by a given point in the plane perpendicular to the incident direction of laser beam can be computed as $S_{h} \times S_{v}$.

Besides sampling space, the included angle between normal direction of foliage elements and incident direction of laser beams will also determine the represented surface area of a given point. The foliage element's orientation can be obtained through reconstructing the normal vector of a given point within a neighbor region (Zheng and Moskal, 2012). Once the foliage element's orientation and the incident direction of laser beam is known, the real foliage element surface area represented by the given point can be obtained using following method. Take the point $P_{i}$ as an example (Fig. 3c), $A P_{i}$ is 
the surface perpendicular to the incident direction of laser beam, while $A R_{i}$ is the real surface represented by a given point in real 3-D space. $\theta_{1}$ is the intersection angel between the $A P_{i}$ and $A R_{i}$, and $\theta_{2}$ is the intersection angle between normal vector of $A R_{i}$ and the incident direction of laser beam. Obviously, $\theta_{1}$ is equal to $\theta_{2}$. Thus the area of $P_{i}$ can be calculated as (eqn. 6 and 7):

$$
\begin{aligned}
& A R_{i}=A P_{i} / \cos \left(\theta_{1}\right) \\
& \theta_{2}=\arccos \left|\frac{\boldsymbol{r}_{i} \cdot \boldsymbol{O P}_{i}}{\left|\boldsymbol{r}_{i}\right| \times\left|\boldsymbol{O P} \boldsymbol{P}_{i}\right|}\right|
\end{aligned}
$$

where $\boldsymbol{O P}_{i}$ is the incident direction of laser beam. Mathematically, a boundary condition $\left(\cos \left(\theta_{1}\right)=0.1\right.$, if $\left.\cos \left(\theta_{1}\right)<0.1\right)$ is set when computing $A R_{i}$ to avoid the infinitely large values as the angle $\theta_{1}$ beyond the $84.26^{\circ}$ in this study.

2.6 Woody-to-total area ratio

After converting each point into its represented surface area, we can compute the parameter WTA $(\psi)$ as (eqn. 8,9 , and 10$)$ :

$$
\begin{gathered}
\psi=\frac{A_{n p}}{A_{p}+A_{n p}} \\
A_{p}=\sum_{i=1}^{N_{p}} A_{\text {random }}(\mathrm{i}) \\
A_{n p}=\sum_{i=1}^{N_{\text {np }}} A_{\text {linear }}(\mathrm{i})
\end{gathered}
$$

Where $A_{p}$ is the total surface area of photosynthetic canopy component; $A_{n p}$ is the total surface area of non-photosynthetic canopy component; $A_{\text {random }}$ (i) and $A_{\text {linear }}$ (i) are the surface areas represented by the i-th point from random and linear classes, 
respectively. $N_{p}$ and $N_{n p}$ are the total number of points from random and linear class, respectively.

\section{Please insert Figure 3 here.}

\section{Results}

\subsection{Point cloud classification}

By conducting the point-wise classification algorithm as described in section 2.4, we obtained the classified PCD of the artificial tree with three different categories including photosynthetic, non-photosynthetic forest canopy components, and bare earth (Fig. 4a4d). Based on the accuracy assessment, our results showed that the non-photosynthetic canopy component (i.e. linear class) held the worst producer's accuracy $(64.91 \%)$, while the producer's accuracy of both photosynthetic canopy component (i.e. random class) and bare earth (i.e. surface class) could reach up to $98.35 \%$ and $99.86 \%$, respectively (Table 3).

In addition, we applied the same classification method to the PCD of an old-growth Douglas fir tree from the WREF site (Fig. 4e), an individual broadleaf tree and a forest plot in BM site (Fig. 4f and 4g), three forest plots PCD in the WPA site (Fig. 4h $-4 j$ ), and an individual broadleaf tree in ND site (Fig. 4k-4m), respectively. There were a lot of grasses existed in the BM site (Fig. $4 \mathrm{f}$ and $4 \mathrm{n}$ ). We only conducted the accuracy assessment for random and linear class used for WTA computation (Table 4). Results from the old-growth tree show a producer's accuracy of random and linear class of $91.85 \%$ and $72.99 \%$, respectively. In the BM site, the producer's accuracy of random and linear class for the individual broadleaf tree are $91.87 \%$ and $78.37 \%$, while the producer's accuracies of random and linear class for the BM forest plot are $96.33 \%$ and 
$78.84 \%$. The producer's accuracy of random and linear classes for the ND individual tree are $98.39 \%$ and $62.05 \%$, the low accuracy of linear class might be attributed to the twigs misclassified as random class. When the effective LAI decreased from 4.15 to 3.13, the producer's accuracy of linear class of the three forest plot PCD increased from $69.43 \%$ to $80.48 \%$, and the user's and producer's accuracy of the random class from forest plot PCD were all more than $80 \%$.

\section{Please insert Figure 4, Table 3 and 4 here.}

\subsection{Point to surface conversion}

By converting the surface area of the individual artificial tree at the single leaf level (Fig. 5a and 5g), woody PCD level (Fig. 5b and 5h), and leaf PCD level (Fig. 5c and 5i) using the proposed method, we found that converted one-side surface area of the individual artificial leaf using the proposed method $\left(1.476 \times 10^{4} \mathrm{~mm}^{2}\right)$ was close to the ones from manual- $\left(1.359 \times 10^{4} \mathrm{~mm}^{2}\right)$ and computer-based $\left(1.350 \times 10^{4} \mathrm{~mm}^{2}\right)($ Table 5). The converted surface area of the leaf and woody PCD of the artificial tree were $5.625 \times 10^{6}$ $\mathrm{mm}^{2}$ and $1.015 \times 10^{6} \mathrm{~mm}^{2}$ using the proposed method. In addition, we obtained the surface area of 'leaf PCD' $\left(5.380 \times 10^{6} \mathrm{~mm}^{2}\right)$ and 'woody PCD' $\left(9.094 \times 10^{5} \mathrm{~mm}^{2}\right)$ using the computer-based mesh method, while the results from manual-based method were $5.081 \times 10^{6} \mathrm{~mm}^{2}$ and $1.001 \times 10^{6} \mathrm{~mm}^{2}$.

In addition, the converted surface areas from partial stem PCD of the old-growth tree from WREF site, the individual broadleaf tree from BM site and the individual broadleaf tree from ND site (Fig. 5d, 5e, and 5f) using the proposed method $(2.688 \times$ $10^{7} \mathrm{~mm}^{2}, 9.849 \times 10^{6} \mathrm{~mm}^{2}$, and $5.437 \times 10^{6} \mathrm{~mm}^{2}$ ) were close to the ones obtained from 
computer-based mesh method $\left(2.514 \times 10^{7} \mathrm{~mm}^{2}, 9.683 \times 10^{6} \mathrm{~mm}^{2}\right.$, and $5.396 \times 10^{6}$ $\mathrm{mm}^{2}$ ) (Fig. 5j, 5k, and 51). To validate the proposed method at forest plot level with smaller leaf sizes, we compared the surface area results of the selected 20 tree stems from BM forest plot obtained using the proposed and computed-based methods. It was found that the results from proposed method can explain $90.26 \%(\mathrm{~N}=20, \mathrm{p}<0.001)$ of the variance in the one obtained using the computer-based method (Fig. 6). Because the surface roughness and curvature of the stem and branches, it is difficult to accurately measure the surface area of woody materials. By comparing the results from proposed method with the one from computer-based method, we found that the accuracy varied from $89.60 \%-98.31 \%$.

\section{Please insert Figure 5 and 6 here.}

In addition, we computed converted woody surface area of the individual artificial tree for classified random (i.e., leaf) $\left(5.910 \times 10^{6} \mathrm{~mm}^{2}\right)$ and linear (i.e., branch and stem) $\left(7.227 \times 10^{5} \mathrm{~cm}^{2}\right)$ class points based on the classified linear class points using point-wise classification method described in section 2.4. The computed WTA based on the converted surface area from 'leaf-on and -off' data, manual-, and computer-based methods were $15.11 \%, 16.44 \%$, and $14.45 \%$, respectively. In the BM site, the WTAs of the individual tree and forest plot computed using the proposed method were $14.83 \%$ and $7.98 \%$, respectively. The old-growth Douglas-fir tree from WREF site held the highest WTA (29.96\%) among all experimental data in this study due to the larger proportion of the woody materials and accumulated forest biomass. However, the WTA for the individual broadleaf tree from ND site is only $4.27 \%$ due to high tree density. As for three forest plot PCD from WPA site, we obtained the WTAs ranging from $7.74 \%$ to 


\section{Please insert Table 6 here.}

In addition, we obtained the PCD for the individual artificial leaf with different sampling space ranging from $2 \mathrm{~mm}$ to $40 \mathrm{~mm}$ with the leaf vertically facing, and at the same height as the TLS (Fig. 7). By doing this, we were able to investigate the effects of sampling space on converted surface area. By scanning and computing the converted surface of an artificial leaf, we found that the converted surface area fluctuated when the sampling space was larger than $8 \mathrm{~mm}$ due to the low level of detailed information about leaf edge. The converted leaf surface area decreased as the sampling space increased from $2 \mathrm{~mm}$ to $8 \mathrm{~mm}$ due to the increasing number of noisy points of leaf edge.

\section{Please insert Figure 7 here.}

\subsection{Direction-based woody-to-total area ratio}

We also computed the WTAs of the individual artificial tree based on each tree PCD obtained from each one of the total five different locations. Before computing the WTA, we removed the replicated points resulting from the registration error by building a 'searching sphere' with each point and predefined sampling space as center and radius, respectively. In addition, we also obtained the classified tree PCD including 'Woody PCD' and 'leaf PCD' for each scan location to compute the WTA. The results showed that the computed WTAs for five different scan locations ranged from $4.39 \%$ to $11.23 \%$ (Table 7), which suggested that the WTA varied with the view direction. This anisotropy of WTA indicates that the WTA computation is a view-direction dependent variable. 


\section{Discussion}

\subsection{Occlusion effects}

Occlusion of foliage elements, which is inherent in all LiDAR acquisitions, resulted from the mutual shading of foliage elements within the forest canopy, which led to incomplete sampling for both photosynthetic and non-photosynthetic canopy components. As a result, the converted surface areas of both photosynthetic and non-photosynthetic canopy components will decrease, and the final WTA results will vary with different spatial distribution patterns of the foliage elements. The increase or decrease in WTA will be determined by the ratio of the converted surface area of photosynthetic and non-photosynthetic canopy components.

One technique to correct for the occlusion effect for this component is achieved by multiplying a correction factor 0.5 (Ma et al., 2016) based on the assumption that typically about $50 \%$ of the tree stem surface under the crown-base height were sampled by one side-lateral TLS scan. Regarding separation of the photosynthetic canopy component and the non-photosynthetic canopy component above crown-base height, Béland et al., (2014) developed an approach to find the occluded voxels with the average size varying from 5 to $200 \mathrm{~cm}$ and corrected for the occlusion effect by assuming a random distribution of foliage elements within it. Since only the geometric information (i.e., $\mathrm{X}, \mathrm{Y}, \mathrm{Z}$ ) is used in the current study, a more sophisticated method should be developed in future research to quantitatively compensate the occlusion effect in terms of the WTA estimation using the laser returned intensity information. In terms of the method proposed by Béland et al., (2014), they used a different TLS with 
dual-channel which can record the high and low laser gain intensity information to classify the photosynthetic and non-photosynthetic canopy components; in addition, an approach aiming to identify the occluded voxels has been developed based on laser gain intensity information. The actual non-random distribution of foliage element of forest canopy (Chen and Cihlar, 1995) doesn't allow to setup the assumption of the azimuthally random distribution of foliage elements.

Due to the occlusion effects between foliage elements existing in both the artificial and real tree, it is difficult to acquire a comprehensive forest PCD even from multiple scanning locations. The missing points resulting from occlusion might change the distribution pattern and further result in misclassification of non-photosynthetic canopy component points. The comparison between WTA estimations of artificial tree PCD obtained from point-wise classification and leaf-on and -off scanning support this explanation. The occlusion of foliage elements also lower the linear class classification accuracy in all three one-central-location-scan forest PCD from WPA site.

\subsection{Classification accuracy effects}

The differences between the converted surface areas using the proposed method and the computer-based approach indicated that the comprehensively sampled tree or forest PCD could be a foundation for further refinement of the final accuracy of TLS-based WTA estimation. Usually, it is very difficult to capture a comprehensive tree or forest PCD even with the multiple scanning locations, especially for the forest canopy with medium or high density (Zolkos et al., 2013). For the artificial tree, the TLS-based WTA estimation will be lower than the true WTA. However, compared with the conventional 
optical instrument-based method, the approach presented here is a 'true' 3-D based approach for estimating WTA. In addition, the separation of photosynthetic and non-photosynthetic canopy components is a key step for TLS-based WTA estimation. Future research along these lines should note that with the changes in forest growth stage, surrounding environment, and climatic condition, WTA will be different. Moreover, the WTA also varies with the azimuth and viewing zenith directions. As has been shown by Zou et al. (2009), the anisotropic characteristic of WTA is a view angle dependent variable. By acquiring the dense forest canopy PCD using TLS, it will enhance the ability to explore the spatial variations of gap fractions with the changes of view zenith angle (Zheng et al., 2016). The view angle dependent forest parameters obtained from TLS can be served as a tool to validate the results obtained from ALS (Aerial Laser Scanning)-based method (Zheng et al., 2016).

Because the point-wise classification method used in this work is based on the geometrical features (i.e., 3-D distribution pattern) of a local point set, the non-photosynthetic canopy component (i.e., branch) affiliated with the photosynthetic canopy component (i.e., leaf) within a local point set region is more easily misclassified as photosynthetic canopy component. Therefore, the classification accuracy of the linear class is usually low among the three different categories which could be justified by the classification results for both individual tree and forest plot PCD in the current study (Table 3 and 4). This limitation could be improved by using different TLS equipment-based classification algorithms (Yang et al., 2013) or by adding the calibrated laser return intensity information. This is a research direction deserving of further exploration. 


\subsection{Searching radius}

The sensitivity analysis on the effects of searching radius on final classification conducted by Ma et al. (2016) suggests that $0.45 \mathrm{~m}$ would be a good choice for forest plots with mid-successional ages $(15-30 \mathrm{yrs}$.). However, in this work, we found that $0.08 \mathrm{~m}$ was an acceptable value for the classification of the individual artificial tree, while a searching radius of $0.75 \mathrm{~m}$ (equaling the $\mathrm{DBH}$ ) was the appropriate value for the old growth tree PCD classification. For the forest PCD in the BM site, searching radius were set as $0.10 \mathrm{~m}$ and $0.30 \mathrm{~m}$ for the forest plot and the individual broadleaf tree which were close to the (average) DBH of tree stem. Moreover, the searching radius was set as $0.35 \mathrm{~m}$ for the individual tree from ND site which is close to its $\mathrm{DBH}$. Thus, DBH of a tree is a good reference value to choose an appropriate searching radius.

\subsection{Sampling space effects}

User predefined sampling space is a key factor in determining the levels of detail captured by the TLS PCD. However, it is not always true that we will get the data with best quality scanned with the finest sampling space with TLS (Ma et al., 2016). The appropriate sampling space should be chosen according to the characteristic size of the foliage element for different tree species. In addition, leaf micro-topography (i.e., leaf wrinkles) will result in the underestimation of converted surface area due to the lack of ability to capturing such fine level of detailed information (Eitel et al., 2014). Lastly, the sampling space for any point of the given forest PCD computed based on the user predefined sampling space should be separately treated for vertical and horizontal aspects.

\subsection{Foliage element orientation effects}


The angle between the incident direction of laser beam and the foliage orientation is another very important variable to determine the converted surface area of a given single point, which will further affect the WTA estimation for an individual tree or forest plot. We computed the converted surface area for the single artificial leaf with and without incorporating the incident direction of laser beam and foliage orientation information. As can been seen in Fig. 8a, the converted leaf surface area decrease substantially as the angle between the foliage orientation and incident direction of laser beams decreases from $90^{\circ}$ to $0^{\circ}$ without considering any angle information for the sampling space. However, the converted surface area results are very close to the actual leaf surface area when the sampling space range is between $2 \mathrm{~mm}$ and $8 \mathrm{~mm}$ (especially around the $5 \mathrm{~mm}$ ) which is the predefined sampling space used for artificial tree scanning in this work (Fig. 8b). Thus, the characteristic size of the foliage elements is a good reference value for the choice of predefined sampling space to obtain the tree or forest PCD using TLS. As for the individual artificial leaf, photosynthetic and non-photosynthetic PCD of the artificial tree, and stems PCD of old-growth Douglas-fir tree, broadleaf tree from ND site, and broadleaf tree from BM site, the good agreement $(89.60 \%-98.31 \%)$ between the converted surface areas and the result obtained from computed-based reconstruction method suggests that the proposed method is feasible and effective to convert the point to surface area for WTA estimation. However, the converted surface area of the old-growth coniferous tree is difficult to compute and validate due to the complex shoot structure by using the convex surface area of each shoot, it might be feasible to improve such estimations through is beyond the scope of this study. The computer-based mesh result is a preferable reference value due to the 
error introduced by the manual measurement based on the cylindrical assumption of tree stem. At the same time, the detailed target object whose characteristic dimension is smaller than the predefined sampling space will be ignored since it is beyond the ability of the TLS instrument to capture such fine-scale variability.

\section{Conclusions}

In this work, we proposed and validated a novel approach to estimate the WTA of an individual tree or forest plot based on a classified tree or forest PCD generated from TLS using a local geometrical feature-based method. By separating the photosynthetic and non-photosynthetic canopy components, a conversion approach was developed to estimate the represented surface area of a given point. We conclude that the incident direction of the laser beam and foliage element orientation are indispensable factors during the estimation of WTA. The user predefined sampling space should be no larger than the characteristic shorter length of the foliage element rectangle bounding box. The proposed method can effectively be used to estimate the WTA of an individual tree or forest plot from 3-D perspective. To obtain reliable WTA estimates, it is recommended that trees should be scanned from multiple scan locations.

\section{Acknowledgement}

Funding and resources for this research project came from the National Science Foundation of China (NSFC) funded Nanjing University (NSFC award \# 41201337) and the NASA Idaho Space Grant Fellowship NNX10AM75H awarded to TSM. This research was conducted at the International Institute for Earth System Science, Nanjing 
University. We also want to thank Nicole Hackman, Jeff Richardson, and Philip Johnsey

for their help in field data collection.

\section{References}

Alonzo, M., Bookhagen, B. and Roberts, D.A., 2014. Urban tree species mapping using hyperspectral and lidar data fusion. Remote Sensing Envir., 148: 70-83.

Amthor, J.S., Gill, D.S. and Bormann, F.H., 1990. Autumnal leaf conductance and apparent photosynthesis by saplings and sprouts in a recently disturbed northern hardwood forest. Oecologia, 84(1): 93-98.

Antonarakis, A.S., Richards, K.S., Brasington, J. and Muller, E., 2010. Determining leaf area index and leafy tree roughness using terrestrial laser scanning. Wat. Resour. Res., 46.

Béland, M., Baldocchi, D.D., Widlowski, J.-L., Fournier, R.A. and Verstraete, M.M., 2014. On seeing the wood from the leaves and the role of voxel size in determining leaf area distribution of forests with terrestrial LiDAR. Agric. For. Met., 184: 82-97.

Brümmer, C. et al., 2012. How climate and vegetation type influence evapotranspiration and water use efficiency in Canadian forest, peatland and grassland ecosystems. Agric. For. Met., 153: 14-30.

Breda, N.J.J., 2003. Ground-based measurements of leaf area index: a review of methods, instruments and current controversies. J. expl Bot., 54(392): 2403-2417.

Calders, K. et al., 2015a. Nondestructive estimates of above-ground biomass using terrestrial laser scanning. Methods in Ecology \& Evolution, 6(2): 198-208.

Calders, K. et al., 2015b. Monitoring spring phenology with high temporal resolution terrestrial LiDAR measurements. Agricultural \& Forest Meteorology, 203: 158-168.

Chasmer, L. et al., 2008. Influences of vegetation structure and elevation on CO2 uptake in a mature jack pine forest in Saskatchewan, Canada. Can. J. For. Res., 38(11): 2746-2761.

Chen, J.M. and Cihlar, J., 1995. Quantifying the effect of canopy architecture on optical measurement of leaf area index using 2 gap size analysis mehtods. IEEE Trans. Geosci. remote Sensing, 33(3): 777-787.

Cook, T.A. and Chakrabarti, S., 2015. Capabilities and performance of dual-wavelength Echidnalidar. Journal of Applied Remote Sensing, 9(1).

Cote, J.F., Widlowski, J.L., Fournier, R.A. and Verstraete, M.M., 2009. The structural and radiative consistency of three-dimensional tree reconstructions from terrestrial lidar. Remote Sensing Envir., 113(5): 1067-1081.

Danson, F.M. et al., 2014. Developing a dual-wavelength full-waveform terrestrial laser scanner to characterize forest canopy structure. Agric. For. Met., 198: 7-14.

Douglas, E.S. et al., 2015. Finding Leaves in the Forest: The Dual-Wavelength Echidna Lidar. Ieee Geoscience and Remote Sensing Letters, 12(4): 776-780.

Eitel, J.U.H., Magney, T.S., Vierling, L.A. and Dittmar, G., 2014. Assessment of crop foliar nitrogen using a novel dual-wavelength laser system and implications for conducting laser-based plant physiology. ISPRS Journal of Photogrammetry and Remote Sensing, 97: 229-240.

Eitel, J.U.H., Vierling, L.A. and Long, D.S., 2010. Simultaneous measurements of plant structure and chlorophyll content in broadleaf saplings with a terrestrial laser scanner. Remote Sensing Envir., 114(10): 2229-2237.

Hosoi, F. and Omasa, K., 2007. Factors contributing to accuracy in the estimation of the woody canopy 
leaf area density profile using 3D portable lidar imaging. J. Exp. Bot., 58(12): 3463-3473.

Kucharik, C.J., Norman, J.M. and Gower, S.T., 1998a. Measurements of branch area and adjusting leaf area index indirect measurements. Agric. For. Met., 91(1-2): 69-88.

Kucharik, C.J., Norman, J.M. and Gower, S.T., 1998b. Measurements of leaf orientation, light distribution and sunlit leaf area in a boreal aspen forest. Agric. For. Met., 91(1-2): 127-148.

Kucharik, C.J., Norman, J.M., Murdock, L.M. and Gower, S.T., 1997. Characterizing canopy nonrandomness with a multiband vegetation imager (MVI). Journal of Geophysical Research-Atmospheres, 102(D24): 29455-29473.

Lalonde, J.F., Vandapel, N., Huber, D.F. and Hebert, M., 2006. Natural terrain classification using three-dimensional ladar data for ground robot mobility. Journal of Field Robotics, 23(10): 839-861.

Lefsky, M.A. et al., 1999. Lidar remote sensing of the canopy structure and biophysical properties of Douglas-fir western hemlock forests. Remote Sensing Envir., 70(3): 339-361.

Lovell, J.L., Jupp, D.L.B., Culvenor, D.S. and Coops, N.C., 2003. Using airborne and ground-based ranging lidar to measure canopy structure in Australian forests. Can. J. remote Sensing, 29(5): 607-622.

Ma, L. et al., 2016. Improved Salient Feature-Based Approach for Automatically Separating Photosynthetic and Nonphotosynthetic Components Within Terrestrial Lidar Point Cloud Data of Forest Canopies. IEEE Trans. Geosci. remote Sensing, 54(2): 679-696.

Magney, T.S. et al., 2014. Assessing leaf photoprotective mechanisms using terrestrial LiDAR: towards mapping canopy photosynthetic performance in three dimensions. New Phytol., 201(1): 344-356.

Norman, J.M., Campbell, G.S., 1989. Canopy structure. In: J.E. R.W. Pearcy, H.A. Mooney and P.W. rundel (Editor), Plant Physiological Ecology. Field Methods and Instrumentation. Chapman and Hall, New York, pp. 301-325.

Raumonen, P. et al., 2013. Fast Automatic Precision Tree Models from Terrestrial Laser Scanner Data. Remote Sensing, 5(2): 491.

Roberts, S.D. et al., 2005. Estimating individual tree leaf area in loblolly pine plantations using LiDAR-derived measurements of height and crown dimensions. For. Ecol. Mgmt, 213(1-3): 54-70.

Shimabukuro, Y.E., Batista, G.T., Mello, E.M.K., Moreira, J.C. and Duarte, V., 1998. Using shade fraction image segmentation to evaluate deforestation in Landsat Thematic Mapper images of the Amazon Region. Int. J. remote Sens., 19(3): 535-541.

Stovall, A.E. and Shugart, H.H., 2014. Quantifying Forest Carbon and Structure with Terrestrial LiDAR, AGU Fall Meeting.

Vandapel, N., Huber, D.F., Kapuria, A. and Hebert, M., 2004. Natural terrain classification using 3-D ladar data, 2004 IEEE International Conference on Robotics and Automation, Vols 1- 5, Proceedings. IEEE International Conference on Robotics and Automation ICRA, pp. 5117-5122.

Whitford, K.R., Colquhoun, I.J., Lang, A.R.G. and Harper, B.M., 1995. Measuring leaf area index in a sparse eucalyp forest - a comparison of estiamtes from direct measurement, hemispherical photography, sunlight trasmittance and allometric regression. . Agric. For. Met., 74(3-4): 237-249.

Yang, X. et al., 2013. Three-dimensional forest reconstruction and structural parameter retrievals using a terrestrial full-waveform lidar instrument (Echidna $\left.{ }^{\circledR}\right)$. Remote Sensing of Environment, 135: 36-51.

Zhao, K. et al., 2015. Terrestrial lidar remote sensing of forests: Maximum likelihood estimates of 
canopy profile, leaf area index, and leaf angle distribution. Agricultural and Forest Meteorology, 209: 100-113.

Zheng, G. et al., 2016. Assessing the Contribution of Woody Materials to Forest Angular Gap Fraction and Effective Leaf Area Index Using Terrestrial Laser Scanning Data. IEEE Trans. Geosci. remote Sensing, 54(3): 1475-1487.

Zheng, G. and Moskal, L.M., 2012. Leaf Orientation Retrieval From Terrestrial Laser Scanning (TLS) Data. IEEE Trans. Geosci. remote Sensing, 50(10): 3970-3979.

Zheng, G., Moskal, L.M. and Kim, S.H., 2013. Retrieval of Effective Leaf Area Index in Heterogeneous Forests With Terrestrial Laser Scanning. IEEE Trans. Geosci. remote Sensing, 51(2): 777-786.

Zolkos, S.G., Goetz, S.J. and Dubayah, R., 2013. A meta-analysis of terrestrial aboveground biomass estimation using lidar remote sensing. Remote Sensing Envir., 128: 289-298.

Zou, J., Yan, G., Zhu, L. and Zhang, W., 2009. Woody-to-total area ratio determination with a multispectral canopy imager. Tree Physiol., 29(8): 1069-1080. 
Table 1. Technical characteristics of terrestrial laser scanner Leica Scan Station 2.

\begin{tabular}{l|l}
\hline \multicolumn{2}{l}{ Single shot time-of-flight measurement } \\
\hline \multicolumn{2}{l}{ Only first return recorded for each laser beam } \\
\hline Scanning speed & $50,000 \mathrm{pts} / \mathrm{sec}$ \\
\hline Field of view & $\begin{array}{l}\text { Horizontal: } 0 \sim 360 \text { degrees } \\
\text { Vertical: }-45 \sim 90 \text { degrees }\end{array}$ \\
\hline Laser beam divergence & $6 \mathrm{~mm}$ at $50 \mathrm{~m}(0.15 \mathrm{~m} \mathrm{rad})$ \\
\hline Laser beam spot size & $4 \mathrm{~mm}$ (FWHH-based) \\
\hline Minimum angle resolution & $6 \mathrm{~mm}$ (Gaussian-based) \\
\hline Wavelength & $0.3 \mathrm{~mm}(3 \mathrm{e}-06$ radians) \\
\hline Detection range & $532 \mathrm{~nm}$ (visible green light) \\
\hline
\end{tabular}


Table 2. Characteristics of trees in different study areas.

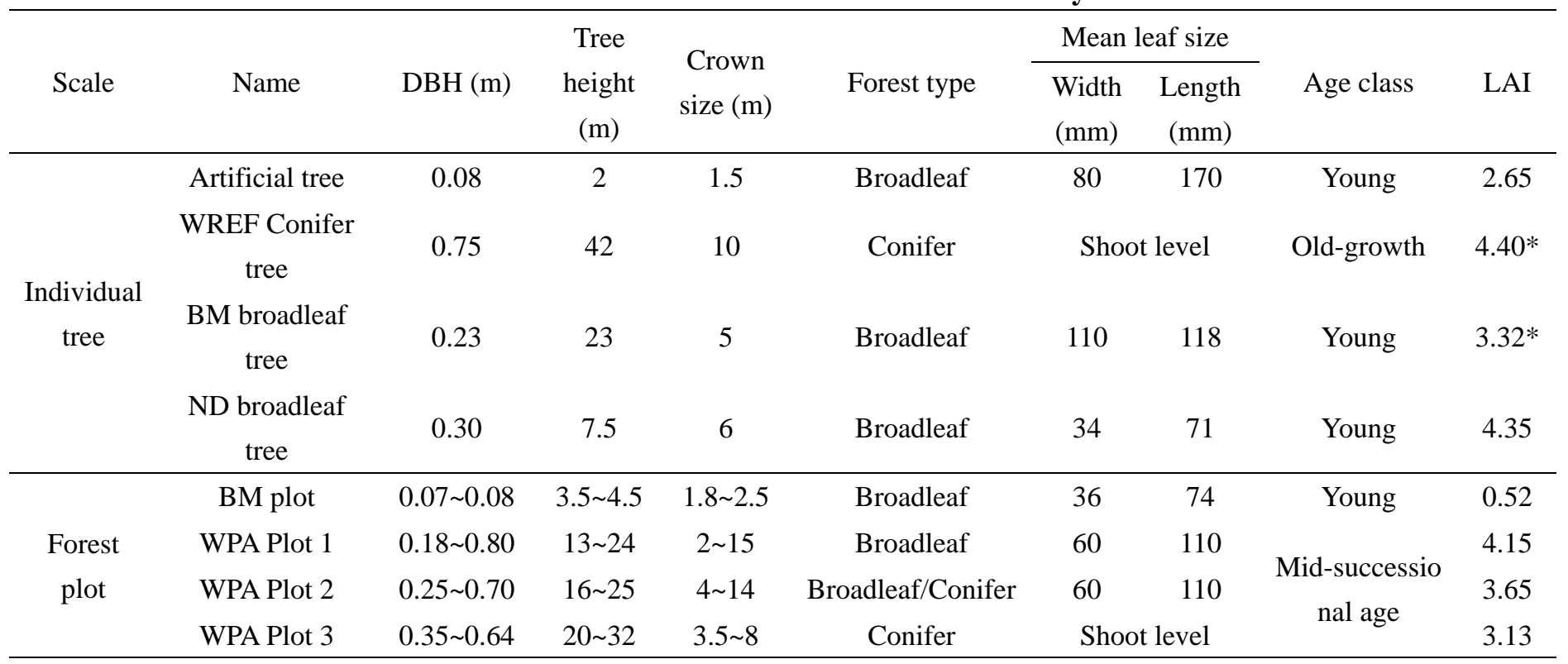

* The values with asterisk represent the LAI measurements for the forest plots. 
Table 3. The classifying accuracy of the artificial tree.

\begin{tabular}{ccccc}
\hline & $\begin{array}{c}\text { Total number } \\
\text { of points }\end{array}$ & $\begin{array}{c}\text { Correctly } \\
\text { classified number } \\
\text { of points }\end{array}$ & $\begin{array}{c}\text { User's } \\
\text { accuracy }\end{array}$ & $\begin{array}{c}\text { Producer's } \\
\text { accuracy }\end{array}$ \\
\hline Surface & 649,724 & 649,573 & $99.98 \%$ & $99.86 \%$ \\
Random & 121,055 & 114,151 & $94.30 \%$ & $98.35 \%$ \\
Linear & 15,886 & 13,053 & $82.17 \%$ & $64.91 \%$ \\
All & 786,665 & 776,777 & $98.74 \%$ & $98.74 \%$ \\
\hline
\end{tabular}


Table 4. The classifying accuracy for the random and linear classes of different individual tree and forest plot PCDs including the old-growth Douglas fir from WREF site, Chinese aspen individual tree and Goldenrain tree forest plots from BM site, Pacific madrone and western Redcedar from WPA site, and Camphor tree from ND site, respectively.

\begin{tabular}{|c|c|c|c|c|c|c|}
\hline & & & $\begin{array}{l}\text { Total } \\
\text { number of } \\
\text { points }\end{array}$ & $\begin{array}{l}\text { Correctly } \\
\text { classified } \\
\text { number of points }\end{array}$ & $\begin{array}{c}\text { User's } \\
\text { accuracy }\end{array}$ & $\begin{array}{c}\text { Producer's } \\
\text { accuracy }\end{array}$ \\
\hline & WRFF & Random & 367,026 & 314,407 & $85.66 \%$ & $91.85 \%$ \\
\hline & old-growth & Linear & 170,208 & 140,460 & $82.52 \%$ & $72.99 \%$ \\
\hline & BM broadleaf & Random & 339921 & 315088 & $92.69 \%$ & $91.87 \%$ \\
\hline & tree & Linear & 105265 & 76725 & $72.89 \%$ & $78.37 \%$ \\
\hline & ND & Random & 1335216 & 1286961 & $96.39 \%$ & $98.39 \%$ \\
\hline & broadleaf tree & Linear & 78619 & 55136 & $70.13 \%$ & $62.05 \%$ \\
\hline & \multirow{2}{*}{ BM plot } & Random & 699356 & 682157 & $97.54 \%$ & $96.33 \%$ \\
\hline & & Linear & 85297 & 62377 & $73.13 \%$ & $78.84 \%$ \\
\hline WPA Plot1 & Random & 784,656 & 650,701 & $82.93 \%$ & $84.07 \%$ & \\
\hline$(\mathrm{LAI}=4.35)$ & Linear & 126,366 & 88,078 & $69.70 \%$ & $69.43 \%$ & \\
\hline WPA Plot2 & Random & 863,945 & 844,308 & $97.73 \%$ & $99.02 \%$ & \\
\hline$(\mathrm{LAI}=3.65)$ & Linear & 191,503 & 140,609 & $73.42 \%$ & $77.10 \%$ & \\
\hline WPA Plot3 & Random & 714,341 & 702,990 & $98.41 \%$ & $99.91 \%$ & \\
\hline$(\mathrm{LAI}=3.13)$ & Linear & 50,406 & 39,799 & $78.96 \%$ & $80.48 \%$ & \\
\hline
\end{tabular}


Table 5. The results of different methods to calculate areas using integrated PCD data of different components for the artificial tree.

\begin{tabular}{cccc}
\hline & Single leaf $\left(\mathrm{mm}^{2}\right)$ & Non-photosynthetic $\left(\mathrm{mm}^{2}\right)$ & Photosynthetic $\left(\mathrm{mm}^{2}\right)$ \\
\hline Manually results & $1.359 \times 10^{4}$ & $1.001 \times 10^{6}$ & $5.081 \times 10^{6}$ \\
Computer mesh results & $1.350 \times 10^{4}$ & $9.094 \times 10^{5}$ & $5.380 \times 10^{6}$ \\
TLS-based results & $1.476 \times 10^{4}$ & $1.015 \times 10^{6}$ & $5.625 \times 10^{6}$ \\
\hline
\end{tabular}


Table 6. The woody-to-total area ratio of different data sets using classification results.

\begin{tabular}{cccc}
\hline & Photosynthetic $\left(\mathrm{mm}^{2}\right)$ & Non-photosynthetic $\left(\mathrm{mm}^{2}\right)$ & WTA $(\%)$ \\
\hline Artificial tree & $5.910 \times 10^{6}$ & $7.227 \times 10^{5}$ & 10.90 \\
Old-growth tree & $1.072 \times 10^{8}$ & $4.588 \times 10^{7}$ & 29.97 \\
BM-individual tree & $6.942 \times 10^{7}$ & $1.209 \times 10^{7}$ & 14.83 \\
ND-individual tree & $2.500 \times 10^{8}$ & $1.117 \times 10^{7}$ & 4.27 \\
BM-plot & $1.894 \times 10^{8}$ & $1.643 \times 10^{7}$ & 7.98 \\
WPA-Plot1 & $3.532 \times 10^{9}$ & $5.303 \times 10^{8}$ & 13.05 \\
WPA-Plot2 & $2.706 \times 10^{9}$ & $4.991 \times 10^{8}$ & 15.57 \\
WPA-Plot3 & $3.370 \times 10^{9}$ & $2.826 \times 10^{8}$ & 7.74 \\
\hline
\end{tabular}


Table 7. Woody-to-total area ratio of each station for the artificial tree using classification results.

\begin{tabular}{cccccc}
\hline & $\mathrm{S} 1$ & $\mathrm{~S} 2$ & $\mathrm{~S} 3$ & $\mathrm{~S} 4$ & $\mathrm{~S} 5$ \\
\hline Leaf area $\left(\mathrm{mm}^{2}\right)$ & $2.215 \times 10^{6}$ & $2.746 \times 10^{6}$ & $2.170 \times 10^{6}$ & $4.607 \times 10^{6}$ & $1.520 \times 10^{6}$ \\
Woody area $\left(\mathrm{mm}^{2}\right)$ & $1.861 \times 10^{5}$ & $1.260 \times 10^{5}$ & $2.122 \times 10^{5}$ & $4.428 \times 10^{5}$ & $1.922 \times 10^{5}$ \\
WTA $(\%)$ & 7.75 & 4.39 & 8.91 & 8.77 & 11.23 \\
\hline
\end{tabular}




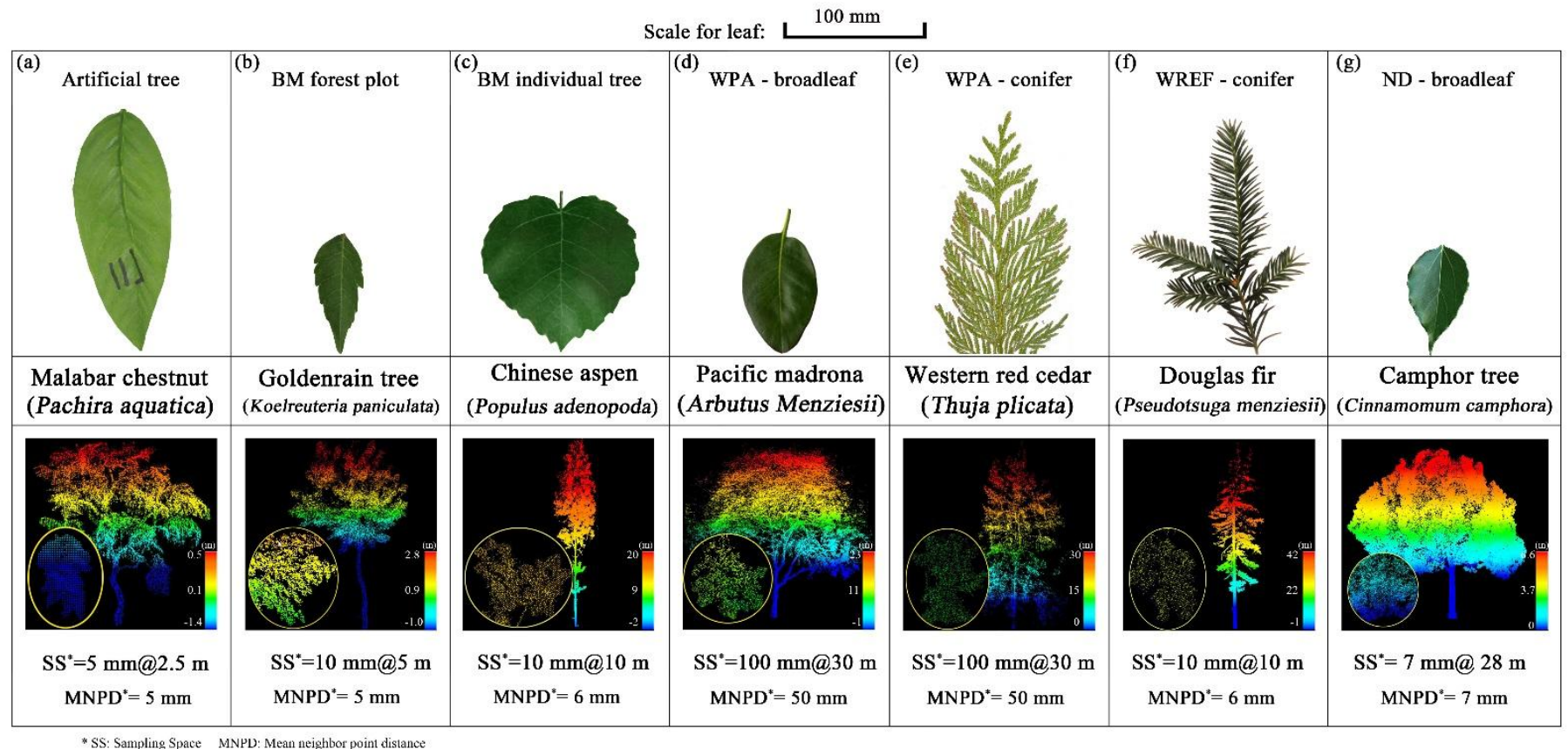

"SS: Sampling Space MNPD: Mean neighbor point distance

Figure 1. Photographs of foliage associated with trees used in this study and the corresponding point cloud data (PCD) and their characteristics including average distance between the scanner and the scanned tree (average distance), sampling space (SS), and mean neighbor point distance (MNPD). The different colors of the point cloud data represent the tree height information. 


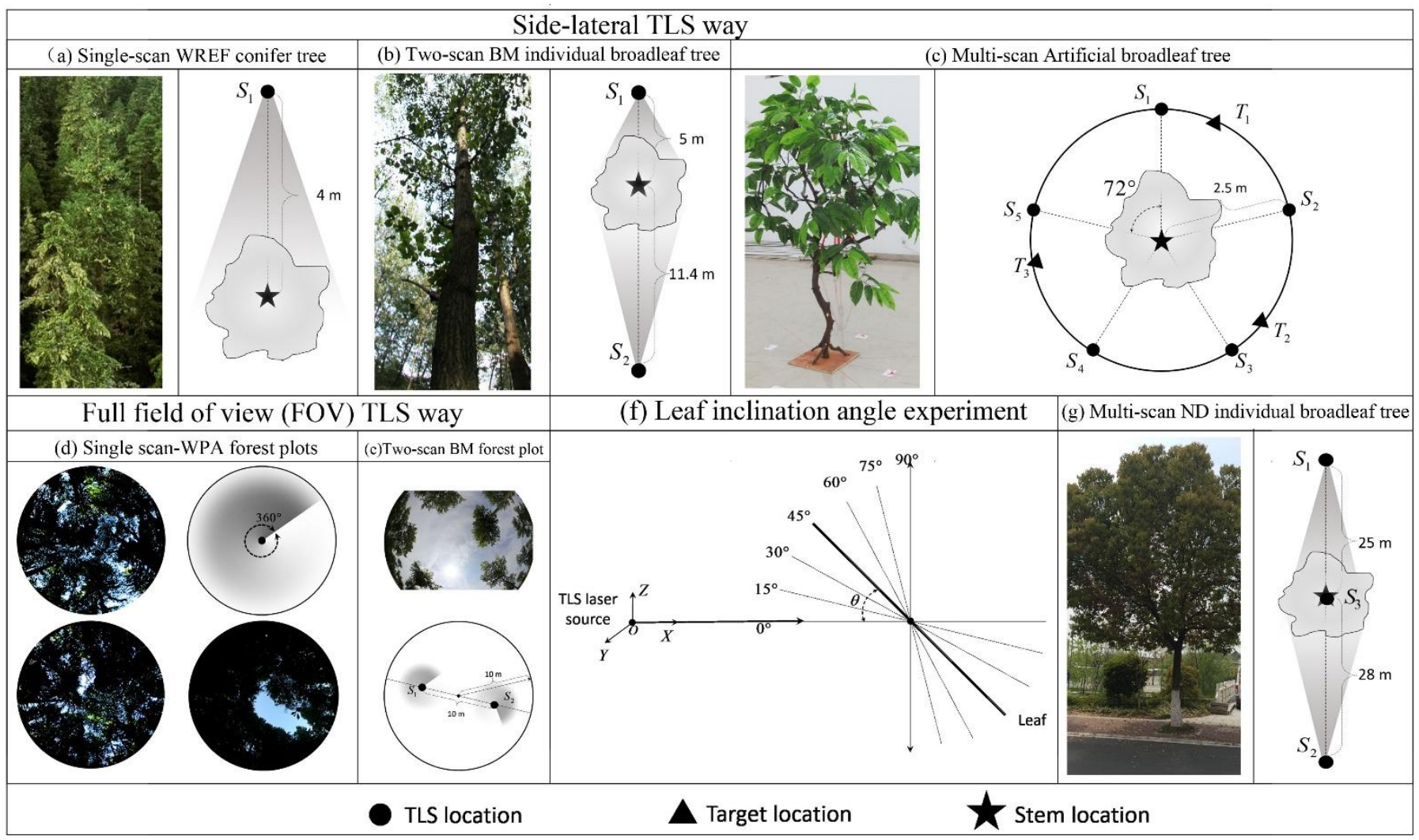

Figure 2. Experimental setup for different individual trees and forest plots. Inset (a) is the bird's eye view (left) of the old-growth Douglas-fir trees in the Wind River Experimental Forest (WREF) site; and the schematic diagram (right) shows the TLS scanner setup; inset (b) is the bottom-up view (left) of the Chinese aspen tree at the BaiMa (BM) experimental forest site and the schematic diagram of the two side-lateral TLS scanner setup; inset (c) is the an image of the artificial tree (left), and the TLS scanner setup (right) for the multi-location TLS acquisition within a well-controlled indoor environment; inset (d) are the digital hemispherical photos (DHP) (leaf and lower right) of three forest plots in the Washington Park Arboretum (WPA) site and the schematic diagram (upper right) to show the one central full FOV (Horizontal: $0^{\circ} \sim 360^{\circ}$, Vertical: $-45^{\circ} \sim 90^{\circ}$ ) TLS scanner setup; inset (e) is the DHP photo for the forest plots in BM site, and the schematic diagram to show the two full-FOV TLS acquisition field setup with the distance between two scan locations and radius of the forest plot $<=10 \mathrm{~m}$; inset (f) is a schematic diagram of the experimental setup for an individual artificial broadleaf with inclination angle changing between $0^{\circ}$ to $90^{\circ}$ in $15^{\circ}$ intervals; and inset (g) is the side-lateral view photo (left) of the medium age Camphor tree in the NanDa campus and a schematic diagram of TLS scanner setup (right). 


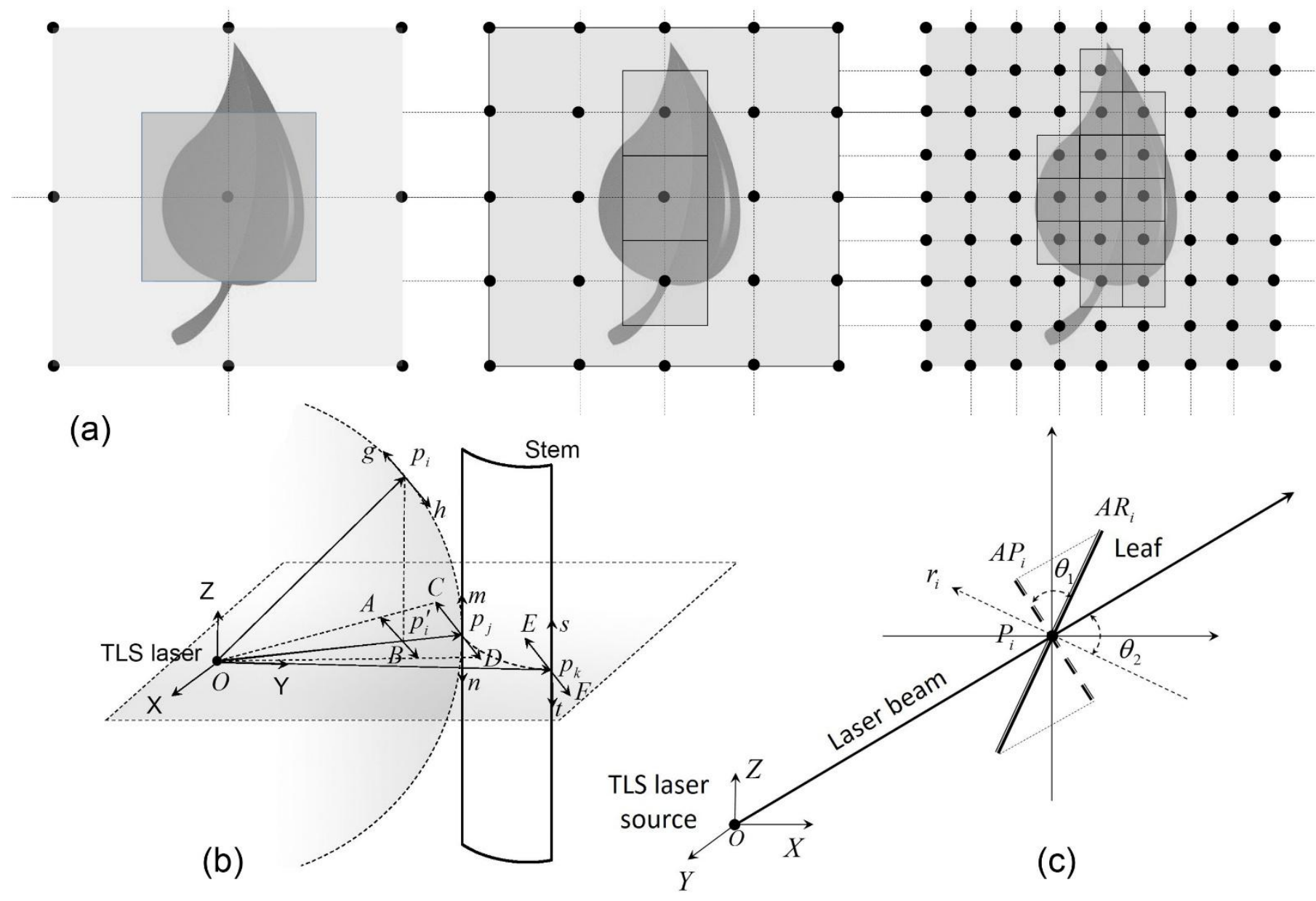

Figure 3. Schematic diagram to illustrate geometrical relationship to compute the horizontal and vertical sampling spacing and convert a single point to surface area with considering the foliage element orientation and incident direction of laser beam. Inset (a) is a schematic diagram to show the sampled points and converted surface areas of a leaf with different sampling spacing, each solid dot represents a point recorded by TLS, and the corresponding square with the dot as center point is the surface area represented by this dot. There are the sampled points with edge as $X, 2 X$, and $4 X$ unit length with the sampling space increasing. In inset $(b), p_{i}$ is a point from the foliage element of a forest canopy, $p_{j}$ and $p_{k}$ are points at the same horizontal surface plane from the stem surface of the tree. $p_{i}{ }^{\prime}$ is the projected point of $\boldsymbol{P}_{i}$ on the same horizontal plane with points $p_{j}$ and $p_{k}$, the vertical sampling spacing are all the same for three different points $(g h=m n=s t)$ since they have the same distances away from TLS. However, the horizontal sampling space of $p_{i}$ is different from the other two points $(A B<C D=E F)$; in inset (c), point $P_{i}$ is any point of a leaf; $A R_{i}$ is the cross-section of a leaf; $r_{i}$ is the normal vector of the plane $A R_{i} ; A P_{i}$ is the projection of $A R_{i}$ in the plane perpendicular to the incident direction of laser beam; $\theta_{I}$ is the intersection angel between $A P_{i}$ and $A R_{i}$, and $\theta_{2}$ is the intersection angle between the incident direction of laser beams and the normal vector of $A R_{i}$. 


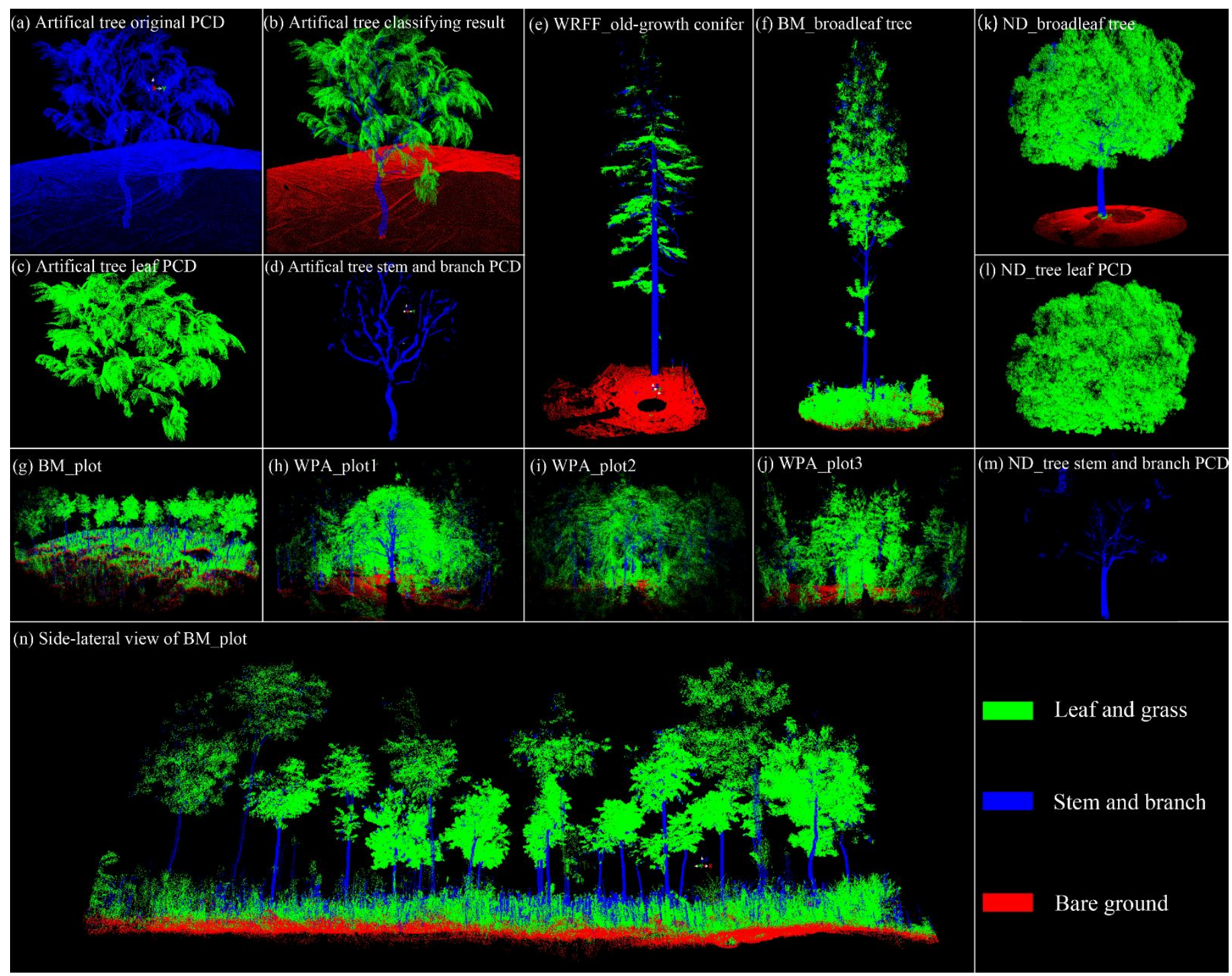

Figure 4. The classification results of individual tree and forest plot PCD. Inset (a) shows the registered artificial tree PCD from five different scanning locations; inset (b) shows the classified artificial tree PCD with three different classes including linear class (i.e. branch and stem) with blue color, surface class (i.e. ground) with red color, and random class (i.e. leaves and grass) with green color; inset (c) and (d) are the isolated points for random and linear classes; inset (e) shows the classification results associated with the old-growth Douglas fir tree PCD from the WREF site; inset (f) and (g) show the classified individual tree and forest PCDs from BM site; insets (h), (i), and (j) show the classified forest PCD for three forest plots from WPA site with the effective LAI as 4.15, 3.65, and 3.13, respectively; and the classified forest PCD for the individual broadleaf tree from ND site including classified tree (k), leaf points (l), and stem and branches points (m), respectively; inset (n) shows the side-lateral view of the BM forest plot with a lot of grass near ground surface. 


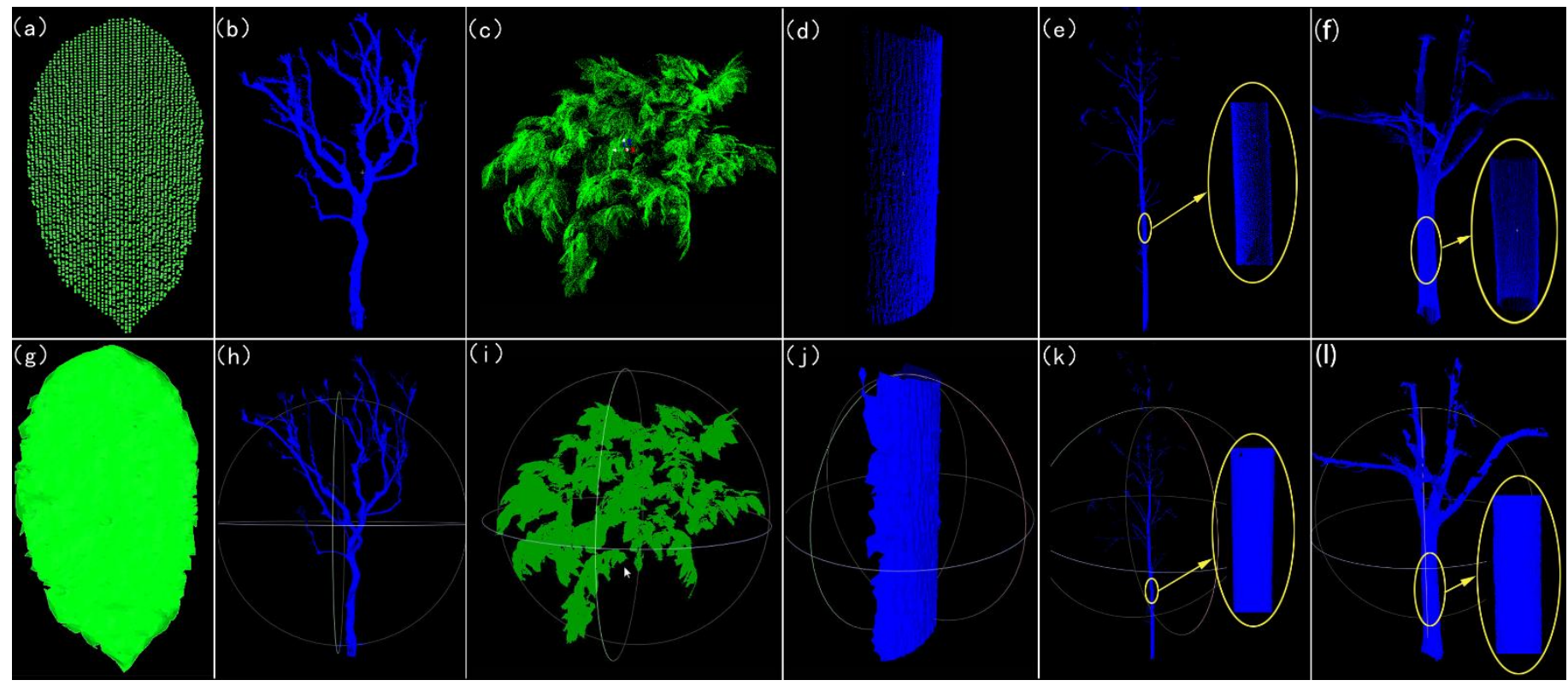

Figure 5. The discrete PCD and corresponding computer-based mesh reconstructing surface results. Inset (a) is the discrete points of an individual artificial leaf and its corresponding reconstructed mesh surface (g); inset (b) and (c) are the discrete stem points of stem and leaves of the artificial tree and their mesh surfaces (h) and (i), respectively; inset (d) is the partial stem surface points of the old-growth Douglas fir tree from the WREF site and its reconstructed surface $(\mathrm{j})$; the inset $(\mathrm{e})$ and $(\mathrm{k})$ are the non-photosynthetic canopy component $\mathrm{PCD}$ and meshed surface with a zoom up view of the individual Chinese aspen tree from the BM site; and the manually classified woody points (f) for the individual tree from ND site, and its computer-based mesh results (l), respectively. 


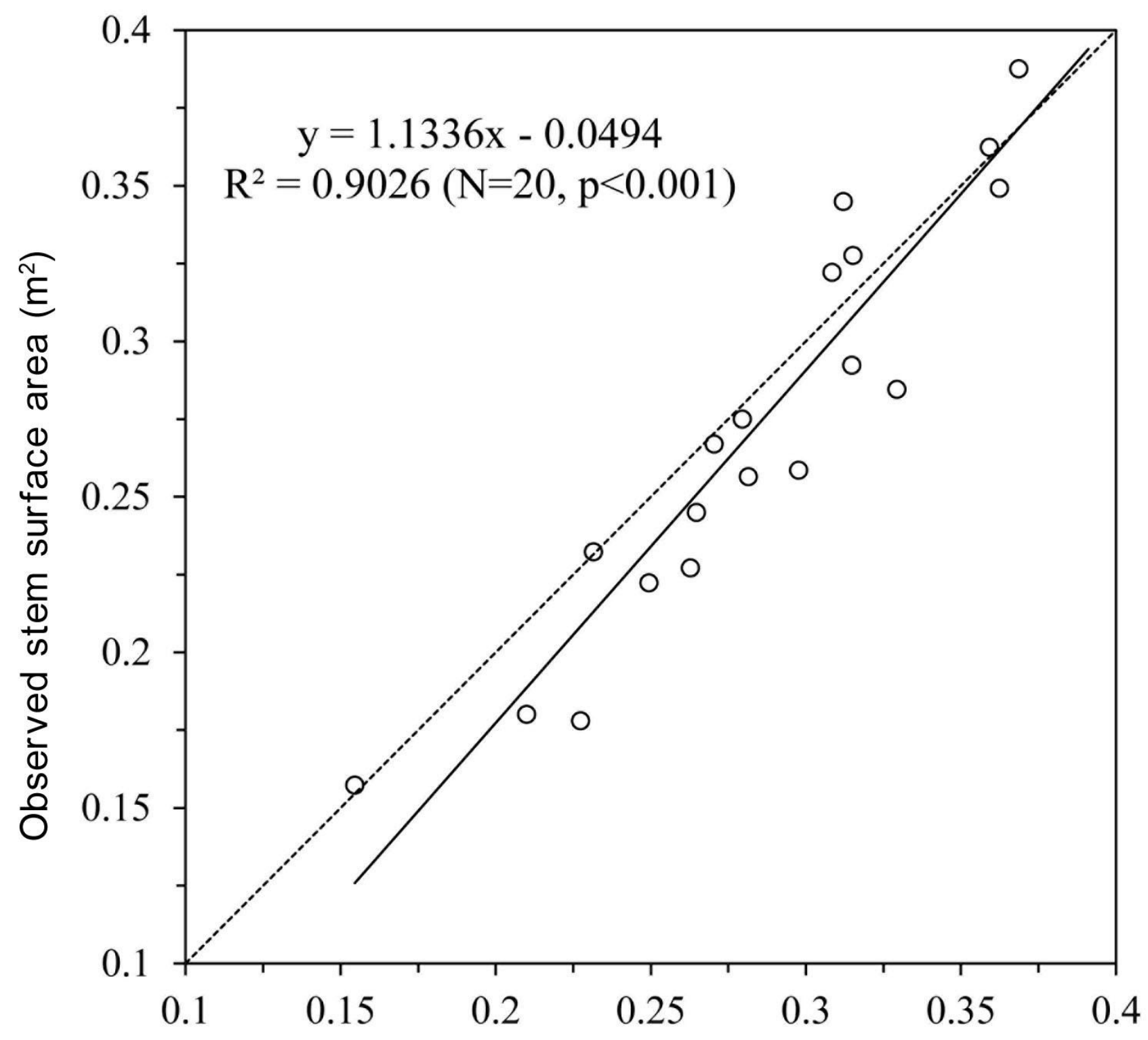

Terrestrial laser scanner derived stem surface area $\left(\mathrm{m}^{2}\right)$

Figure 6. The stem surface area comparison between computer-based mesh results and the calculated stem surface area using the proposed method for the twenty tree stems from the forest plot PCDs in BM site. 


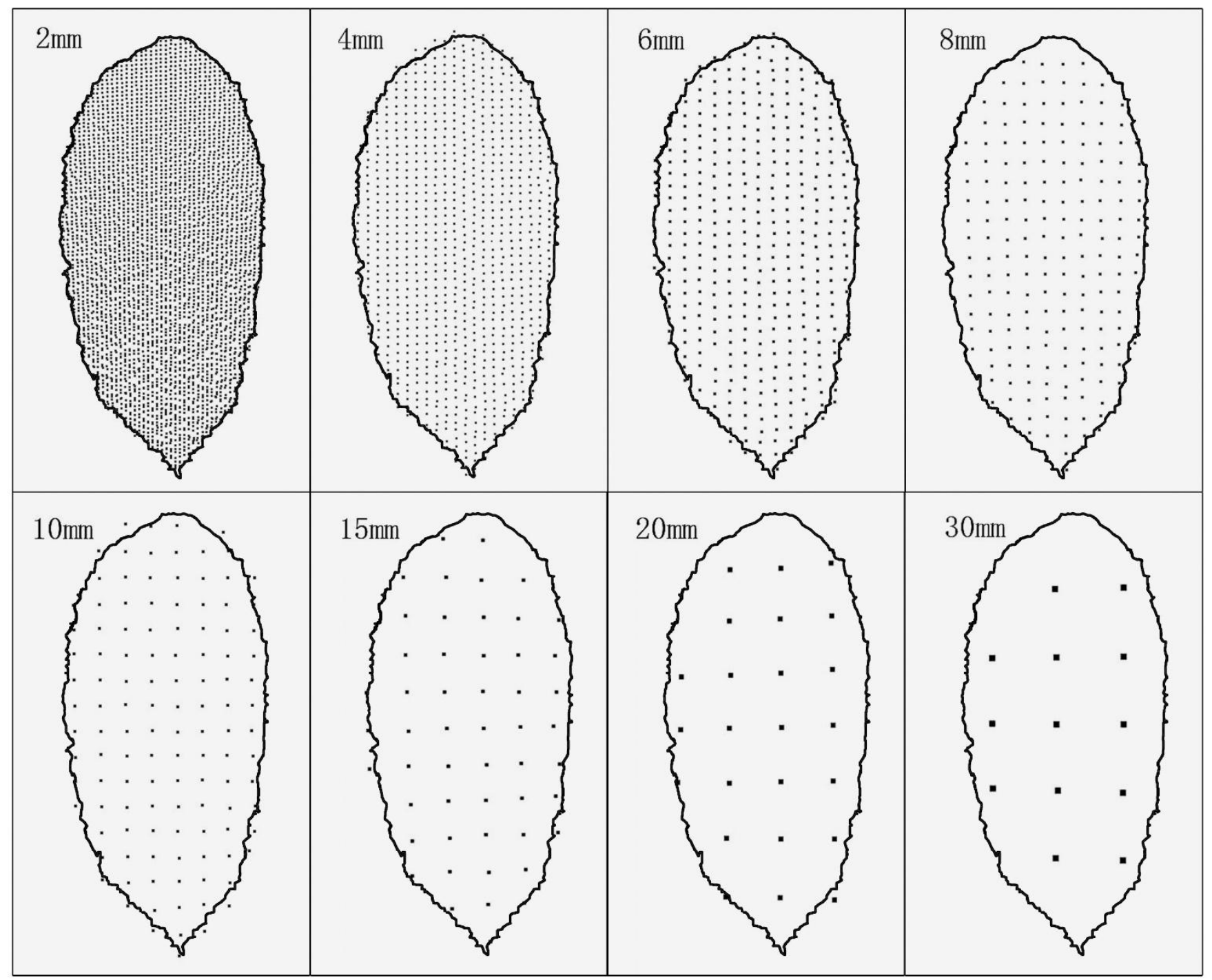

Figure 7. The different distribution patterns of sampled points for an individual artificial leaf with the same height setup between the laser origin point and center point of the artificial leaf vertically facing to TLS. 


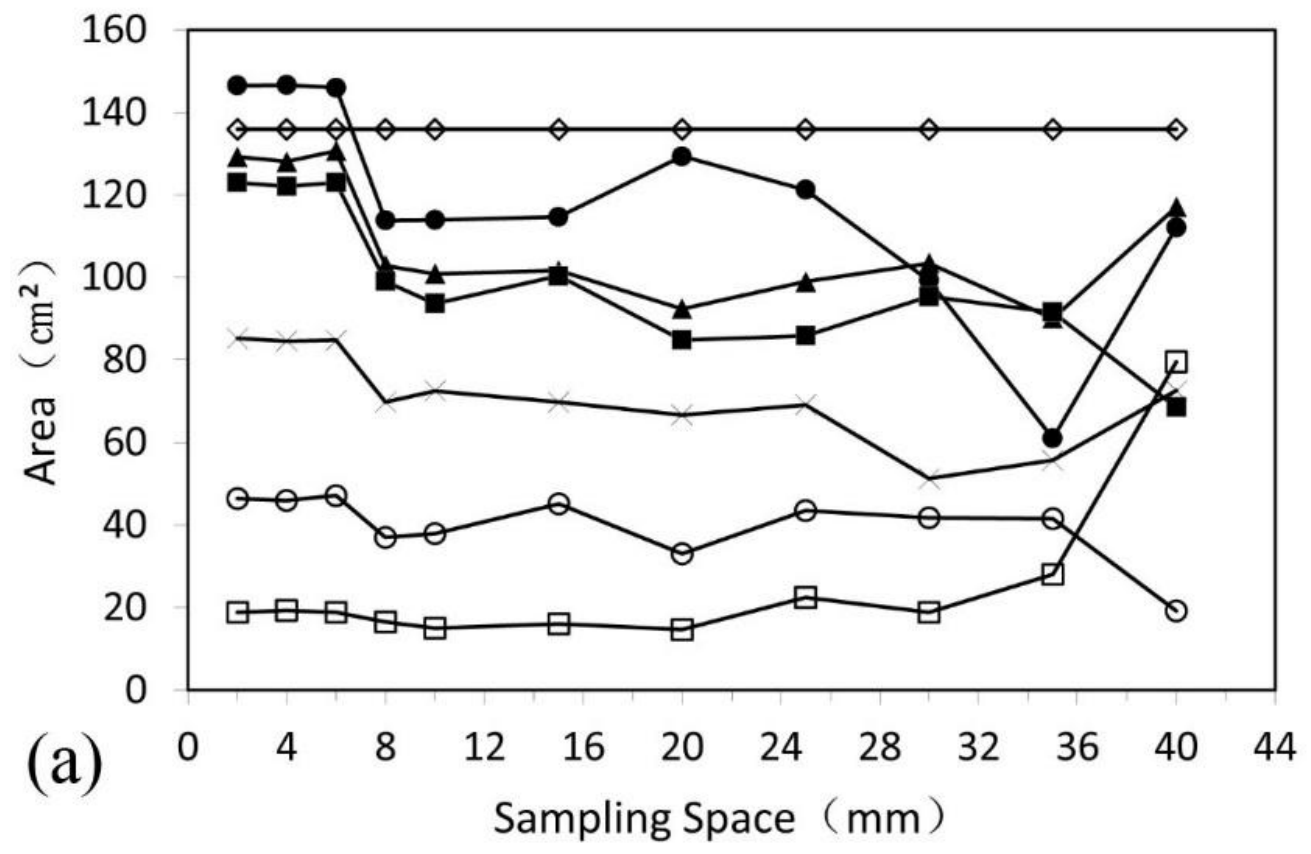

$\rightarrow 90^{\circ} \rightarrow 75^{\circ} \rightarrow-60^{\circ} \longrightarrow 45^{\circ} \bigcirc-30^{\circ} \square-15^{\circ} \diamond$ True_Value

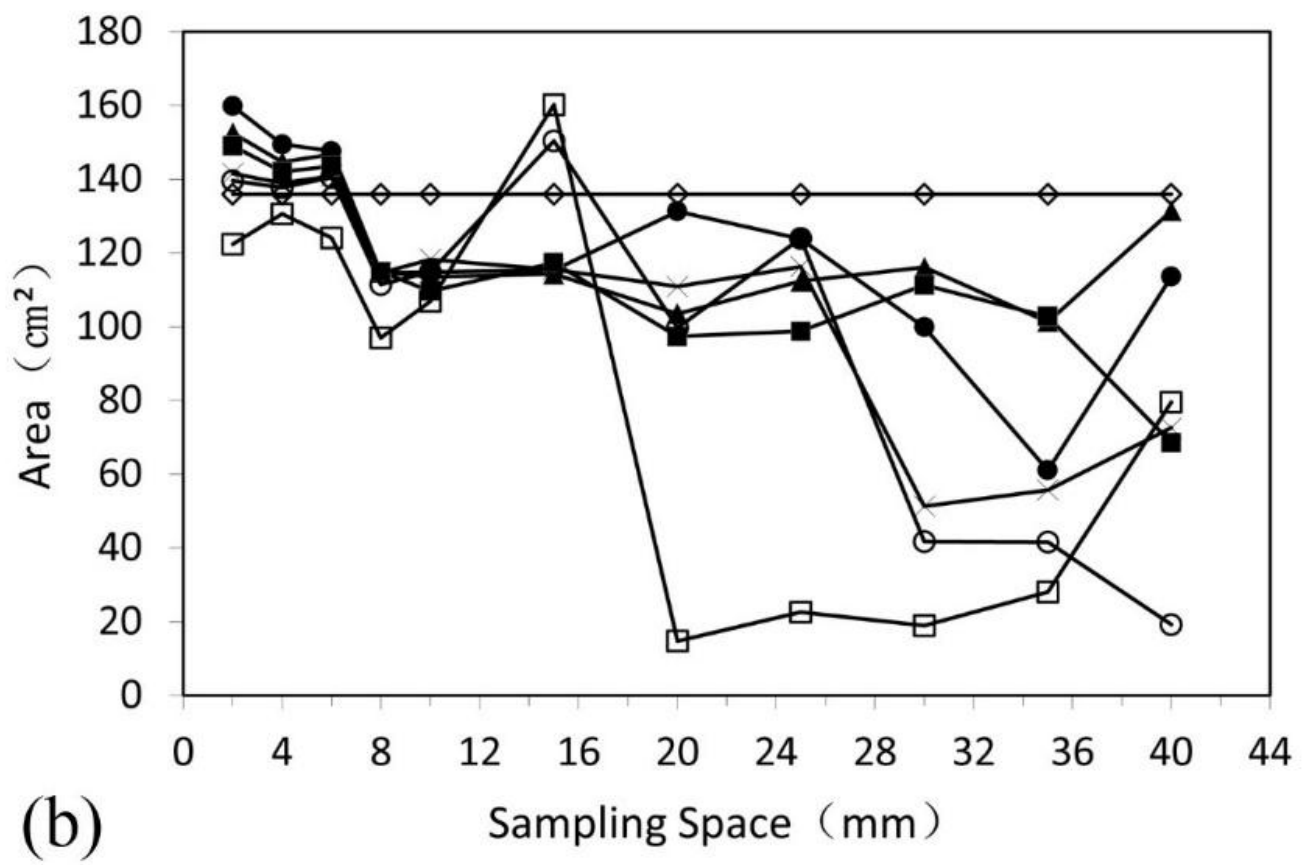

$\multimap-90^{\circ} \longleftarrow 75^{\circ} \rightarrow-60^{\circ} \longrightarrow 45^{\circ} \multimap-30^{\circ} \square-15^{\circ} \multimap-T r u e$ Value

Figure 8. The converted represented surface areas by a single point of the artificial leaf without (a) and with (b) considering the incident direction of laser beam and foliage orientation for various predefined sampling space setups. 